\title{
Nonlinear Disturbance Observer Based Robust Tracking Control of Pneumatic Muscle
}

\author{
Youssif Mohamed Toum Elobaid, ${ }^{1,2}$ Jain Huang, ${ }^{1}$ and Yongji Wang ${ }^{1}$ \\ ${ }^{1}$ School of Automation, Huazhong University of Science and Technology, No. 1037 Luoyu Road, Hongshan District, \\ Wuhan, Hubei 430074, China \\ ${ }^{2}$ Department of Control Engineering, University of Alneelain, Gamhoria Street, 11121 Khartoum, Sudan
}

Correspondence should be addressed to Jain Huang; huang_jan@mail.hust.edu.cn

Received 4 December 2013; Accepted 9 February 2014; Published 20 March 2014

Academic Editor: Huaicheng Yan

Copyright (C) 2014 Youssif Mohamed Toum Elobaid et al. This is an open access article distributed under the Creative Commons Attribution License, which permits unrestricted use, distribution, and reproduction in any medium, provided the original work is properly cited.

\begin{abstract}
Presently pneumatic muscles (PMs) are used in various applications due to their simple construction, lightweight, and high force-to-weight ratio. However, pneumatic muscles are facing various problems due to their nonlinear characteristics and various uncertainties in real applications. To cope with the uncertainties and strong nonlinearity of a PM model, a nonlinear disturbance observer (NDO) is designed to estimate the lumped disturbance. Based on the disturbance observer, the tracking control of PM is studied. Stability analysis based on Lyapunov method with respect to our proposed control law is discussed. The simulation results show the validity, effectiveness, and enhancing robustness of the proposed methods.
\end{abstract}

\section{Introduction}

Designing a robot arm that performs tasks in anthropic environments in a fashion similar to a human arm is the goal of many ongoing research projects. Task-oriented rehabilitation therapy is becoming exciting as an important issue and needs to be addressed in a sufficient way. In particular, safe and not inducing further injury or pain during motor function training need to be carefully investigated since they are the main challenges in such kind of task $[1,2]$. Owing to the absence of compliance in robot, pneumatic muscle actuators (PMAs) are considered to be basic actuators and offer the advantage of intrinsic elasticity to achieve joint compliance [3]. A PMA is an interesting actuator, which is very similar to animal skeletal muscle action in size, weight, and power output. It works in a similar manner to human or animal muscles. There are several advantages of PMA, such as lower cost, light weight, compliance, and very high power/weight and power/volume ratios. These ratios are about five times higher in comparison to an electric motor or a hydraulic actuator [4].

However, all that glitter is not gold. The slower response in force-generation and nonlinear parameters depending on the load, position, and speed of PMAs compared to electric motors is the main problem making pneumatic actuators inaccurate and difficult to control $[4,5]$. To overcome this complexity and hindrance, in recent years a great effort in human-friendly robotic systems has focused on the development of actuation systems that can provide robots with the characteristics of safety, accuracy, ease to control, and high performance. So far, the researchers presented a number of novel approaches to alleviate the design of controller in practical applications of PMA. Repperger et al. modelled the PMA as a stiffness-visco model consisting of a spring element and damping element arranged in parallel [6]. Reynolds et al. proposed and constructed an improved new phenomenological model, which is consisting of a contractile element, a spring element, and a damping element based on Repperger's model [7]. With a progress in the area of networked robotic systems interact closely with humans [8]; researchers have investigated nonlinear control methods, with attention to safety requirements as well as the traditional metrics of performance. Accordingly, there are an abundance of researches on safe robots and new results on stability and $H_{\infty}$ performance have been developed to help humans $[9,10]$. 
Xing et al. presented a wearable exoskeletal robot for upper extremity stroke rehabilitation called "RUPERT" which has four actuated degrees of freedom driven by compliant and safe pneumatic muscles (PMs) on the shoulder, elbow, and wrist [9]. A nonlinear control strategy as a cascaded tracking control concept for pneumatic muscle actuators is presented in [11]. The main objective is to control a trolley, which is driven by an artificial muscle to follow a reference path.

To improve the control performance of the PM, Xing et al. have driven a new type of actuator, which comprises a pneumatic muscle (PM) and a torsion spring; in this study, the sliding mode approach is applied to the tracking control problem of a planar arm manipulator system $[12,13]$.

In [14-16] a fuzzy logic control has been used to overcome poor models, the approach is not mathematical and mimics how a person would make decisions. Emanuel Todorov et al. have worked with pneumatically actuated robots and reported results on modeling and control of a 2-DOF robot, as well as preliminary results on a state-of-the-art 38-DOF humanoid [17]. Varga and Moučka have presented results gained in research experiments with an artificial muscle; the realized experiments were focused on finding the exact mechanical features of artificial muscle, which will be used for development of a theoretical model. This model can be later used for direct control of the muscle [18].

It is found that using a disturbance observer can further improve the robustness of nonlinear control system. Recently, some works have been presented about the observer design for nonlinear systems [1, 12, 19-21].

This study proposes a Nonlinear Disturbance Observer Based Control (NDOBC) approach for the PM system. A major advantage and novelty of our framework is that it allows us to develop in a separate way the control law from the observer design provided that each part satisfies some stability properties. In general, the main objective of the use of a disturbance observer is to deduce the external unknown or uncertain disturbance without the use of an additional sensor. Friction is a very common phenomenon in mechanical systems and plays an important role in system performance. Many friction models and compensation methods have been proposed, one of the most promising methods is Nonlinear Disturbance Observer Based Control (NDOBC) where a NDO is used to estimate the friction.

To cope with the uncertainties and strong nonlinearity of the pneumatic muscle a nonlinear disturbance observer (NDO) is presented in this paper. Lyapunov concepts are the fundamental tools invoked to analyze the closed-loop PM behavior which leads, by carefully selecting the observer gain function, to the stability and utilization of asymptotic position tracking performance. Stabilization controller is designed by using Lyapunov theory on the pneumatic muscle system, and then the observer design theory is used for interference suppression under the condition of existing model error and external disturbance. The scheme enhances the robustness of the position tracking and improves the tracking accuracy.

PID controllers are one of the most used types of controllers in practice. They are easy to realize and can stabilize a system even without knowing the model. However, in spite of the simplicity and the small number of parameters that have to be adjusted, it is hard to analyze the stability and tune the parameters when using PID controllers. Therefore, the major significance of the proposed Nonlinear Disturbance Observer Based Control (NDOBC) controller lies in its high robustness against disturbance and superior performance over the conventional PID controller (see Figure 10). Moreover, NDOBC's engineering implementation is also easy, which explores a convenient engineering method to improve the performance of the PM control system.

The layout of this paper is as follows. In Section 2, we derive the model of the PM system. Section 3 concerns with Nonlinear Disturbance Observer Based Control design. Section 4 discusses the stability analysis. Simulation results are presented and discussed in Section 5. We finally end by the conclusion in Section 6.

\section{System Model}

PMAs are assumed as actuators in many applications, where their static and dynamic characteristics play an important role in the overall behavior of the control system. Therefore, improving the dynamic behavior of the pneumatic muscle actuator is of prime interest to control system designers.

Two main categories for the mathematical models of a pneumatic muscle actuator are prevalent: the theoretical and the phenomenological models. The theoretical models, which are derived from the law of energy conservation, describe PMA behavior based on quasistatic states without inclusion of explicitly temporal information. However, this approach limits its application for real-time control because not only it is too complex in structure, but also it requires too many parameters that are difficult to obtain during experimentation $[1,22,23]$.

In this paper, we adopt the phenomenological model as a combination of effects from nonlinear friction, spring, and contraction components to describe the dynamic behavior of a pneumatic muscle (PM) pulling a mass against gravity as in Figure 1(b).

The coefficients related to these three elements depend on the input pressure of the PM [1]. The equations describing approximately the dynamics of a PM are given by

$$
\begin{gathered}
M \ddot{x}+B(P) \dot{x}+K(P) x=F(P)-M g, \\
K(P)=K_{0}+K_{1} P, \\
B(P)=B_{0 i}+B_{1 i} P \quad \text { (inflation), } \\
B(P)=B_{0 d}+B_{1 d} P \quad \text { (deflation), } \\
F(P)=F_{0}+F_{1} P,
\end{gathered}
$$

where $M$ is the mass, $g$ is the acceleration of gravity, $\Delta x=0$ corresponds to the fully deflated position (see Figure 1(a)), and $P$ is the input pressure. The coefficients $K(P)$ and $B(P)$ are pressure dependent for the spring and the damping, respectively. The contractile element presented the effective 


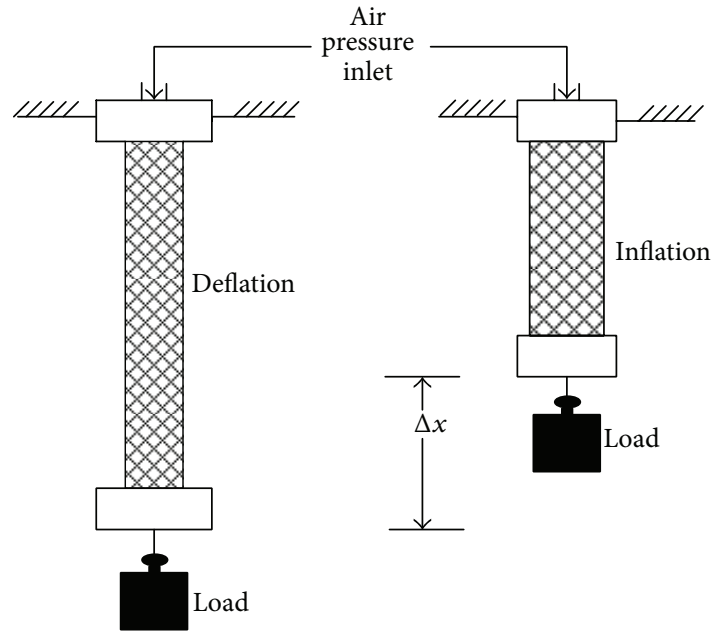

(a)

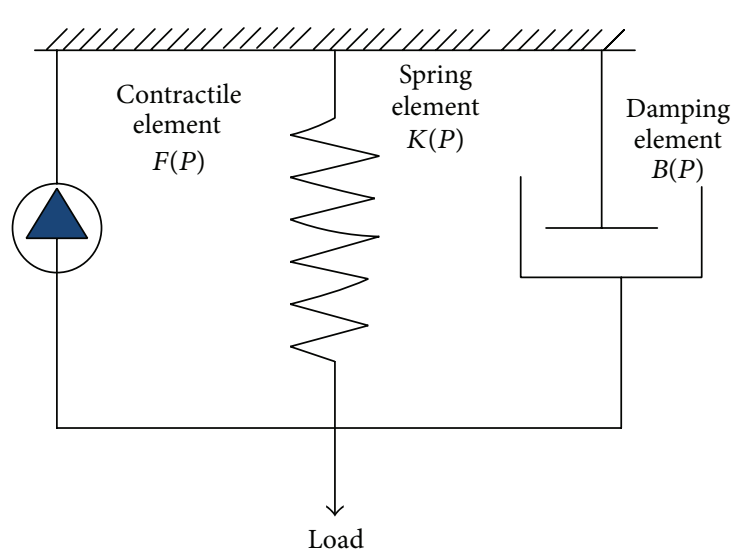

(b)

Figure 1: (a) The operational principle of a PM; (b) the three-element model of PM.

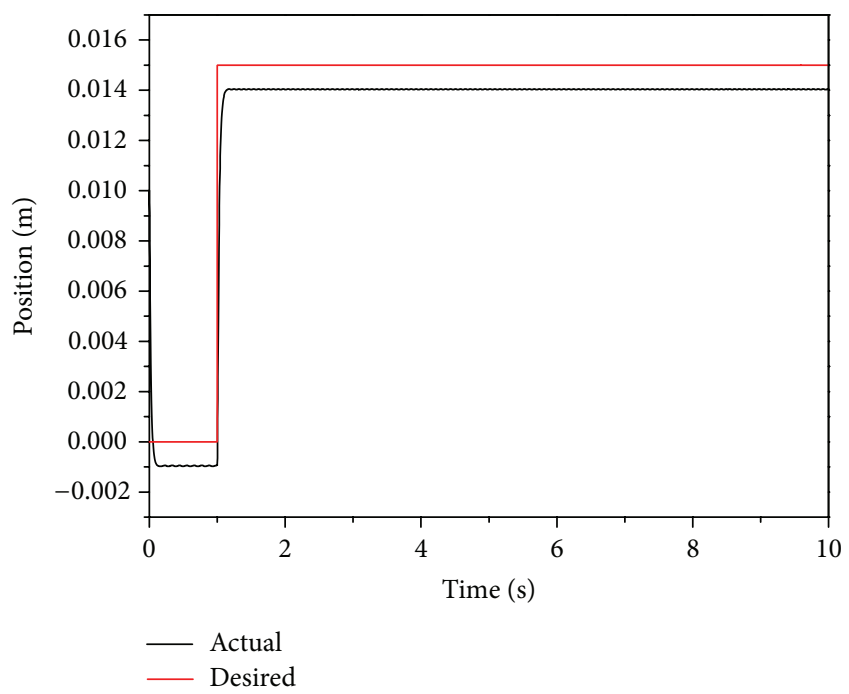

FIGURE 2: Step-trajectory tracking result without NDO.

force $F(P)$. The damping coefficient depends on whether the $\mathrm{PM}$ is inflated and deflated. From the dynamic equations (1)(5), we can write for the PM the following state model:

$\dot{q}_{1}=q_{2}$,

$\dot{q}_{2}=\frac{1}{M}\left[\left(F_{0}-M g-B_{0} q_{2}-K_{0} q_{1}\right)+\left(F_{1}-B_{1} q_{2}-K_{1} q_{1}\right) P\right]$,

with state variable $\left[q_{1}, q_{2}\right]^{T}=[x, \dot{x}]^{T}$. In summary, the dynamics model of PM can be concretely represented as

$$
M \ddot{x}+B_{0} \dot{x}+K_{0} x+\left(M g-F_{0}\right)=\left(F_{1}-B_{1} \dot{x}-k_{1} x\right) P .
$$

Let us define $e=x-x_{r}, \dot{e}=\dot{x}-\dot{x}_{r}$, and $\ddot{e}=\ddot{x}-\ddot{x}_{r}$ where $e, \dot{e}$ and $\ddot{e}$ are the position, speed, and acceleration tracking

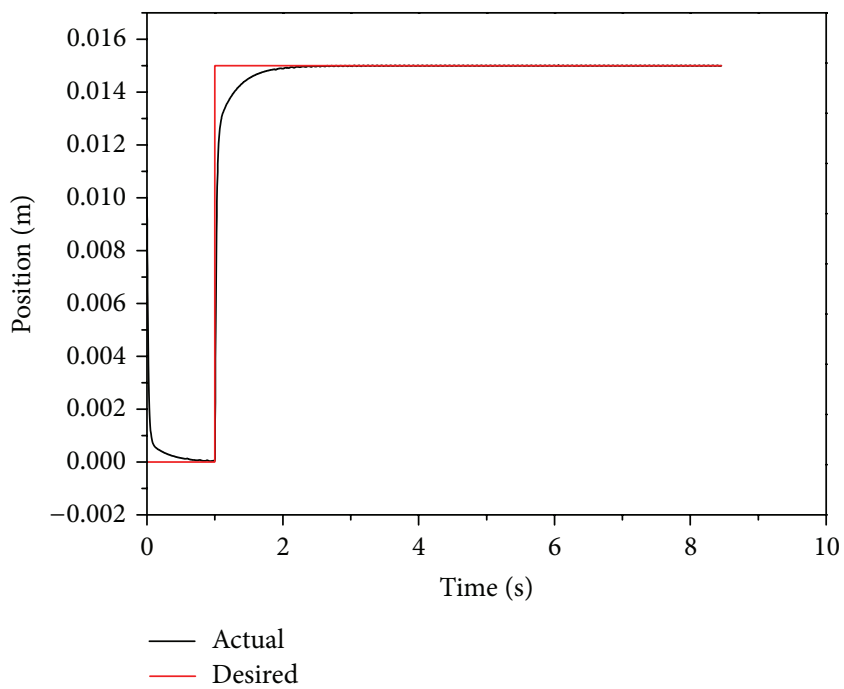

FIGURE 3: Step-trajectory tracking result with NDO.

error, respectively. Substituting these errors in (7) then we have error-differential equation satisfying the following:

$$
\begin{aligned}
M \ddot{e}+ & M \ddot{x}_{r}+B_{0} \dot{e}+B_{0} \dot{x}_{r}+K_{0} e+K_{0} x_{r}+\left(M g-F_{0}\right) \\
& =\left(F_{1}-B_{1} \dot{e}-B_{1} \dot{x}_{r}-K_{1} e-K_{1} x_{r}\right) P .
\end{aligned}
$$

Let us introduce an additional control component $\nu$ satisfying

$$
\begin{aligned}
\left(F_{1}-\right. & \left.B_{1} \dot{e}-B_{1} \dot{x}_{r}-K_{1} e-K_{1} x_{r}\right) P \\
& =M \ddot{x}_{r}+B_{0} \dot{x}_{r}+K_{0} x_{r}+\left(M g-F_{0}\right)-K_{P} e+\nu, \\
\nu & M \ddot{e}+B_{0} \dot{e}+\left(K_{0}+K_{P}\right) e=M \ddot{e}+B_{0} \dot{e}+\beta e,
\end{aligned}
$$

where $\beta=K_{0}+K_{P}$ is a constant. 


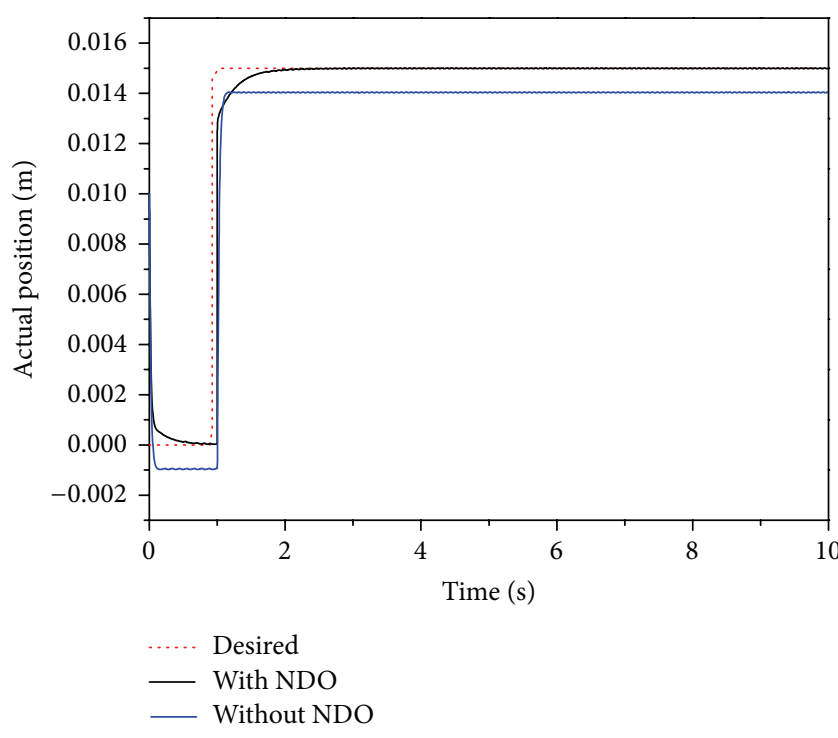

FIGURE 4: Step-trajectory comparison for position tracking with/without NDO.

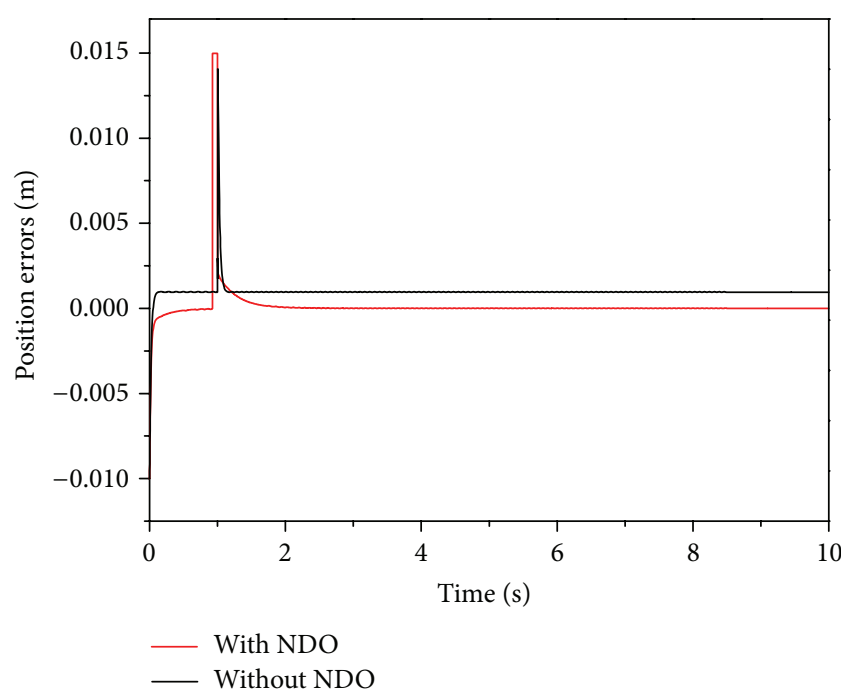

FIGURE 5: Step-input tracking control error (amplitude $=15 \mathrm{~mm})$.

Let us define another state variable satisfying $\left[x_{1}, x_{2}\right]^{T}=$ $[e, \dot{e}+e]^{T}$. Then the error dynamic model in (10) can be translated into the following equation of state:

$$
\begin{aligned}
\dot{x}_{1}= & -x_{1}+x_{2}, \\
\dot{x}_{2}= & \frac{1}{M}\left(\left(B_{0}-K_{0}-K_{P}-M\right) x_{1}-\left(B_{0}-M\right) x_{2}-H_{0}\right) \\
& \quad+\frac{H_{1}}{M} P,
\end{aligned}
$$

where $H_{0}=M \ddot{x}_{r}+B_{0} \dot{x}_{r}+K_{0} x_{r}+\left(M g-F_{0}\right)-K_{P} e$ and $H_{1}=F_{1}-B_{1} \dot{e}-B_{1} \dot{x}_{r}-K_{1} e-K_{1} x_{r}$.

\section{Nonlinear Disturbance Observer (NDO) Based Control Design}

In this section, we discuss the Nonlinear Disturbance Observer Based Robust Control (NDOBRC) approach for PM-tracking control. Estimation of nonlinear uncertainty can be pursued through generating the uncertainties as the output of some exogenous system, allowing estimation and compensation under certain conditions. It is a matter of fact that a model of the exosystems must be included into the controller to reach the design goals. Owing to this, a composite controller must be built consisting of two parts: a controller without or having poor disturbance attenuation ability and a disturbance observer.

As the dynamics of a PM is nonlinear and hard to model precisely, the design of the model-based control algorithm is more cumbersome. Besides the modeling uncertainties, external disturbances are inevitable in real environments which degrade the control performance. Therefore, the controller should have a robust capability to achieve the desired objective. Since the PM model shows highly nonlinear behavior, it is difficult to estimate the proper norm bound and, thus, the usual robust control method for PM control often results in a conservative design. Therefore, the use of a disturbance observer resolves these difficulties.

Considering the external disturbances $\omega$ and the model error in (11) the general PM model with interference is given by

$$
\dot{X}=f(X)+g_{1}(X) P+g_{2}(X) \omega,
$$

where

$$
\begin{gathered}
\dot{X}=\left[\begin{array}{ll}
\dot{x}_{1} & \dot{x}_{2}
\end{array}\right]^{T}, \quad g_{1}(X)=\left[\begin{array}{ll}
0 & \frac{H_{1}}{M}
\end{array}\right]^{T}, \\
g_{2}(X)=\left[\begin{array}{ll}
0 & \frac{1}{M}
\end{array}\right]^{T},
\end{gathered}
$$

$f(X)$

$$
=\left[\begin{array}{ll}
-x_{1}+x_{2} & \frac{1}{M}\left(\left(B_{0}-K_{0}-K_{P}-M\right) x_{1}-\left(B_{0}-M\right) x_{2}-H_{0}\right)
\end{array}\right]^{T} .
$$

To estimate the disturbance $\omega$ a nonlinear disturbance observer is proposed as

$$
\dot{\widehat{\omega}}=\alpha\left(\dot{X}-f(X)-g_{1}(X) P-g_{2}(X) \widehat{\omega}\right)
$$

with $\alpha=\left[\begin{array}{ll}c_{1} & c_{2}\end{array}\right]$ where $c_{1}>0$ and $c_{2}>0$.

Let us define observer residuals as $\widetilde{\omega}=\omega-\widehat{\omega}$; then the observer in (14) can be given by

$$
\begin{aligned}
\dot{\hat{\omega}} & =\alpha\left(\dot{X}-f(X)-g_{1}(X) P-g_{2}(X) \widehat{\omega}\right) \\
& =\alpha \cdot g_{2}(X)(\omega-\widehat{\omega})=\left[\begin{array}{ll}
c_{1} & c_{2}
\end{array}\right] g_{2}(X) \widetilde{\omega} .
\end{aligned}
$$

Since $g_{2}(X)=\left[\begin{array}{ll}0 & 1 / M\end{array}\right]^{T}$ then we have $\dot{\hat{\omega}}=c_{2} \widetilde{\omega} / M$.

Define an auxiliary vector $z=\widehat{\omega}-\rho(X)$ where $z \in R^{2}$, $\rho(X)$ is a nonlinear function to be designed, and the nonlinear 


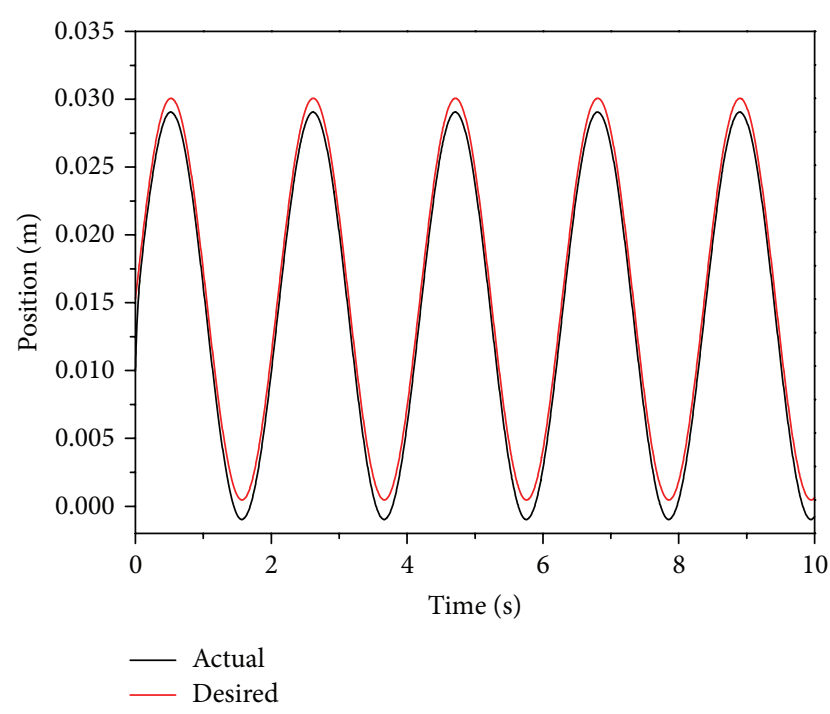

FIGURE 6: Sinusoidal-trajectory tracking result without NDO.

observer gain $\alpha$ is defined as $\alpha=\partial \rho(X) / \partial X$. Taking the time derivative of $z$ and substituting (14) give us

$$
\dot{z}=-\alpha\left(f(X)+g_{1}(X) P+g_{2}(X)(z+\rho(X))\right) .
$$

Substituting $\alpha$ in (16) the disturbance observer can be deduced as

$$
\begin{gathered}
\widehat{\omega}=z+\rho(X), \\
\dot{z}=-c_{1}\left(-x_{1}+x_{2}\right)-\frac{c_{2}}{M} \\
\times\left(\left(B_{0}-K_{0}-K_{P}-M\right) x_{1}-\left(B_{0}-M\right) x_{2}-H_{0}+H_{1} P\right) \\
-\frac{c_{2}}{M}\left(z+c_{1} x_{1}+c_{2} x_{2}\right),
\end{gathered}
$$

where $\rho(X)=c_{1} x_{1}+c_{2} x_{2}$.

Considering (9) and (12), the Nonlinear Disturbance Observer Based Robust Control (NDOBRC) law can be given by

$$
P=\frac{M \ddot{x}_{r}+B_{0} \dot{x}_{r}+K_{0} x_{r}+\left(M g-F_{0}\right)-K_{P} e+v-\widehat{\omega}}{F_{1}-B_{1} \dot{e}-B_{1} \dot{x}_{r}-K_{1} e-K_{1} x_{r}} .
$$

\section{Stability Analysis}

The main focus will be on control design based on the statespace model for a given PM. There is typically some freedom in the choice of Lyapunov function for the control design; we call $V$ a Lyapunov function if, in a certain neighborhood of the equilibrium point, $V$ is positive definite and its derivative along the system trajectories is negative semidefinite. The main Lyapunov stability results for PM control systems can be summarized by the following lemma and theorem.

Lemma 1 (see [24]). If, in a ball $B_{R 0}$ around the equilibrium point 0 , there exists a scalar function $V(x, t)$ with continuous partial derivatives such that

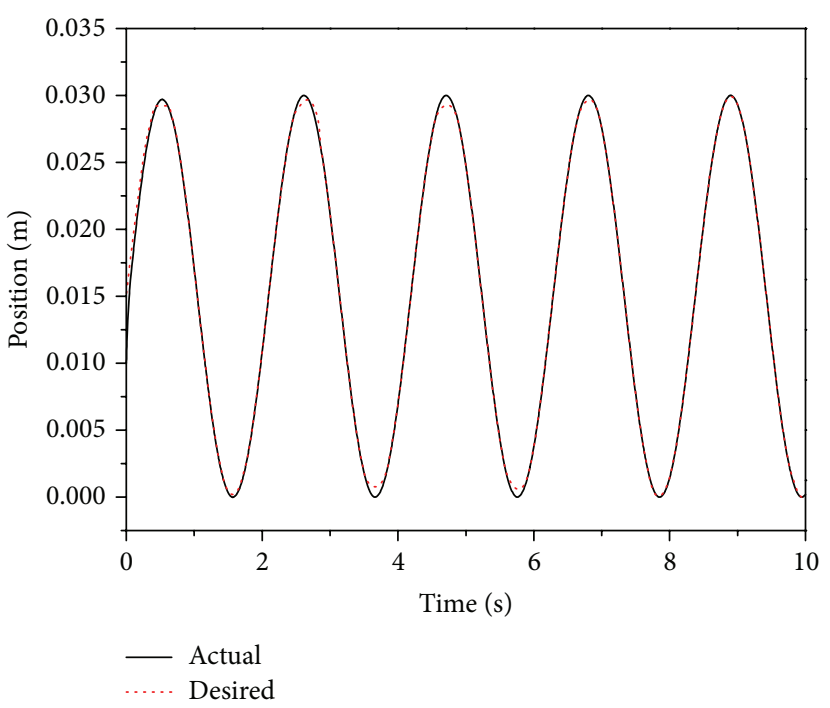

FIGURE 7: Sinusoidal-trajectory tracking result with NDO.

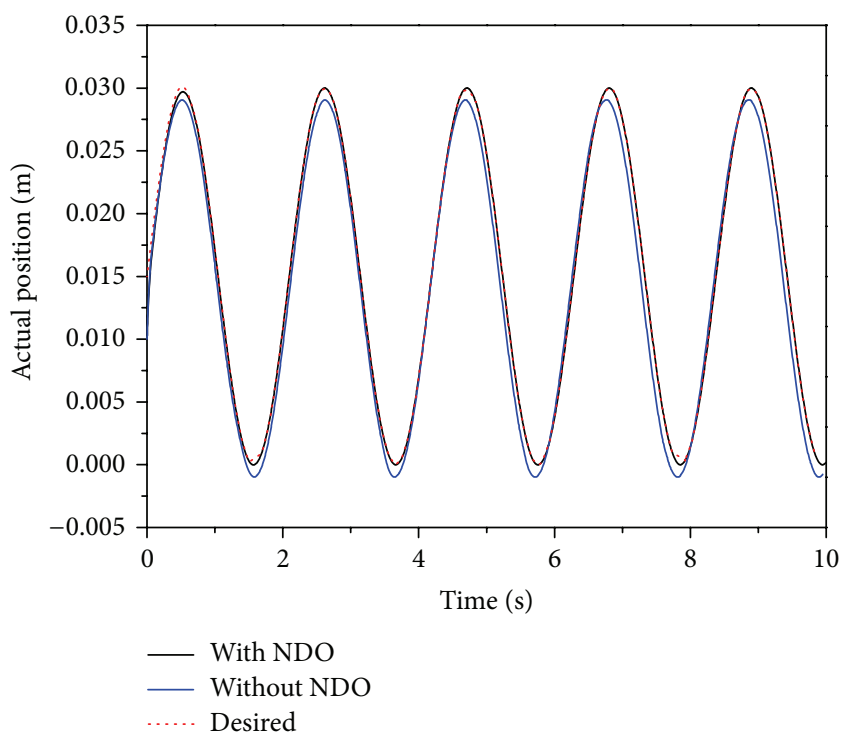

FIGURE 8: Sinusoidal-trajectory comparison for position tracking with/without NDO.

(a) $V$ is positive definite

(b) $\dot{V}$ is negative semidefinite

then the equilibrium point 0 is stable in the sense of Lyapunov. If, furthermore,

(c) $V$ is decrescent

then the origin is uniformly stable. If condition $(b)$ is strengthened by requiring that $V$ be negative definite, then the equilibrium point is uniformly asymptotically stable.

If the ball $B_{R}$ is replaced by the whole state space, and condition ( $a$ ), the strengthened condition (b), condition (c), and the condition

(d) $V(x, 0)$ is radially unbounded 


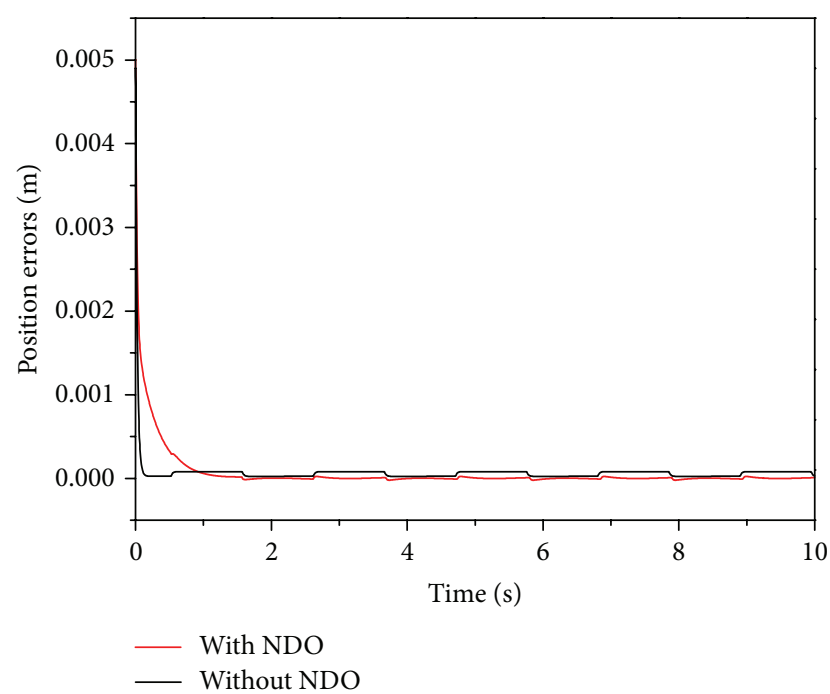

FIGURE 9: Sinusoidal-input tracking control error.

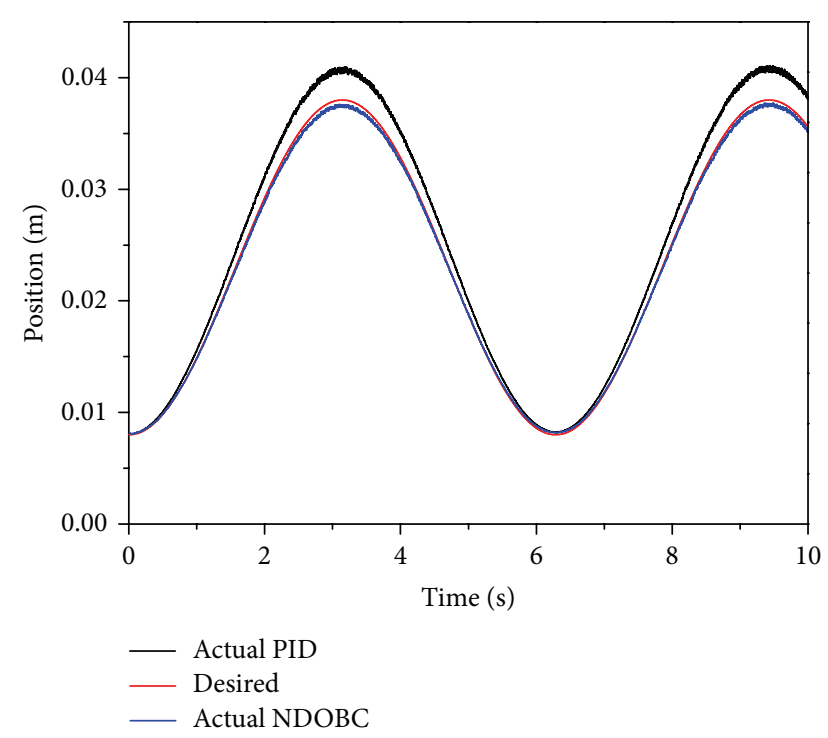

FIGURe 10: A comparison between NDOBC and conventional PID controller.

are all satisfied, then the equilibrium point at 0 is globally uniformly asymptotically stable.

Generally in $\widetilde{\omega}=\omega-\widehat{\omega}$, there is no prior information about the derivative of the disturbance $\omega$. When the disturbance varies slowly relative to the observer dynamics, it is reasonable to suppose that $\dot{\omega}=0$, and then we have $\dot{\tilde{\omega}}=-\dot{\hat{\omega}}$.

Theorem 2. Consider the pneumatic muscle system (1) with a NDOBRC controller (18). Assume that the disturbance $\omega$ varies slowly relative to the observer dynamics. Then, the trajectory of system (1) can be driven asymptotically onto the desired trajectory.
Proof. Consider a positive-definite Lyapunov function $V(t)$ of the form

$$
V=\frac{1}{2} \dot{e}^{T} M \dot{e}+\frac{1}{2} e^{T} \beta e+\frac{1}{2} \widetilde{\omega}^{2} .
$$

By differentiating both sides of (19) we have

$$
\dot{V}=\dot{e}^{T} M \ddot{e}+e^{T} \beta \dot{e}+\widetilde{\omega} \dot{\tilde{\omega}} .
$$

From (10) we can write

$$
M \ddot{e}=\nu-B_{0} \dot{e}-\beta e .
$$

Substituting (21) in (20) $\dot{V}$ can be deduced as

$$
\begin{aligned}
\dot{V} & =\dot{e}^{T}\left(\nu-B_{0} \dot{e}-\beta e\right)+e^{T} \beta \dot{e}+\widetilde{\omega} \dot{\tilde{\omega}} \\
& =\dot{e}^{T} \nu-\dot{e}^{T} B_{0} \dot{e}+\widetilde{\omega} \dot{\tilde{\omega}} \leq \dot{e}^{T} \nu+\widetilde{\omega} \dot{\tilde{\omega}} .
\end{aligned}
$$

Using $\dot{\tilde{\omega}}=-\dot{\widehat{\omega}}=-c_{2} \widetilde{\omega} / M$ in (22) we get

$$
\begin{gathered}
\dot{V} \leq \dot{e}^{T} v+\widetilde{\omega} \dot{\tilde{\omega}}=\dot{e}^{T} v-\frac{c_{2} \widetilde{\omega}^{2}}{M}, \\
\dot{V} \leq \dot{e}^{T} v .
\end{gathered}
$$

Consider Lemma 1 and the simple control law for the additional control component $v$ with a real and positive constant $K$ satisfying the condition $v=-K \dot{e}$ with $K>0$. The Lyapunov function is decreasing. This completes the proof.

Considering (18) with $v=-K \dot{e}$, our Lyapunov control law-based disturbance observer can be deduced as

$$
P=\frac{M \ddot{x}_{r}+B_{0} \dot{x}_{r}+K_{0} x_{r}+\left(M g-F_{0}\right)-K_{P} e-K \dot{e}-\widehat{\omega}}{F_{1}-B_{1} \dot{e}-B_{1} \dot{x}_{r}-K_{1} e-K_{1} x_{r}} .
$$

Substituting (24) in (17) and using $x_{1}=e, x_{2}=\dot{e}+e$ the nonlinear disturbance observer can be deduced as

$$
\begin{gathered}
\widehat{\omega}=z+\rho(X), \\
\dot{z}=-\left[c_{1} \dot{e}+\frac{c_{2}}{M}\left(-\beta e-\left(B_{0}+K-M\right) \dot{e}\right)\right] \\
=\left(\frac{c_{2}}{M}\left(B_{0}+K-M\right)-c_{1}\right) \dot{e}+\frac{c_{2} \cdot \beta}{M} e \\
=\alpha_{1} \cdot \dot{e}+\alpha_{2} \cdot e,
\end{gathered}
$$

where $\beta=K_{0}+K_{P}, \alpha_{1}=\left(c_{2} / M\right)\left(B_{0}+K-M\right)-c_{1}$, and $\alpha_{2}=\left(c_{2} \cdot \beta\right) / M$.

\section{Simulation Results and Discussion}

The simulation has been undertaken in MATLAB/ SIMULINK. The simulation objective is to reveal the strength of the proposed controller. In our simulation, we let the load $M=0.829 \mathrm{~kg}$; the parameters $K$ and $K_{P}$ have been selected as 700 and 500, respectively. The PM coefficients used for the simulation are shown in Table 1. 
TABLE 1: PM coefficient sets used for the simulation.

\begin{tabular}{lccc}
\hline Coefficient & $C$ (Evaluation) & $C_{1}(0.9 \times C)$ & $C_{2}(1.1 \times C)$ \\
\hline$F_{0}\left(\times 10^{2}\right)$ & -1.0336 & -0.9302 & -1.137 \\
$F_{1}\left(\times 10^{-6}\right)$ & 719.75 & 647.78 & 791.73 \\
$K_{0}\left(\times 10^{4}\right)$ & 1.5010 & 1.351 & 1.651 \\
$K_{1}\left(\times 10^{-2}\right)$ & -5.703 & -5.133 & -6.2733 \\
$B_{0}$ & 52.08 & 46.87 & 57.29 \\
$B_{1}\left(\times 10^{-6}\right)$ & -124.5 & -112.1 & -137.0 \\
\hline
\end{tabular}

The parameters $F(P), K(P)$, and $B(P)$ of columns $C_{1}$ and $C_{2}$ are chosen assuming $\pm 10 \%$ error in the evaluation values $(C)$; the simulation interval time was selected as $10 \mathrm{~ms}$.

The exogenous disturbance in our simulation system was considered as Coulomb and viscous friction. When we use our proposed Nonlinear Disturbance Observer based Robust Control (NDOBRC), the external nonlinear disturbance can be approximately estimated by this nonlinear disturbance observer (NDO). The performance of the proposed controller was assessed by setting a step and a sinusoidal-trajectory tracking problem.

Using amplitude of $0.015 \mathrm{~m}$ of step response, in Figure 2 we show the representations of the position with respect to time without integrating the nonlinear disturbance observer (NDO) in the controller. As we can see from the figure, the curves obtained from the proposed controller tend to the desired position after small number of fluctuations. This is due to the fact that the system needs time before reaching the stability point.

When using our proposed NDOBRC method, the influence of disturbances is estimated by a disturbance observer and then compensated for the controller. Therefore, the effects of disturbances and uncertainties are reduced significantly, as depicted in Figure 3. As we can see in this figure, a more satisfactory tracking performance can be achieved.

Figure 4 shows a comparison of control efforts with/without the NDO, as we can see in this figure, the maximum effort was made near the peaks. This is an expected result due to the high rate of change in the peak which makes the disturbance effect of the system stronger. Also the figure indicates that the controller with NDO leads to more accurate tracking performance compared to the controller without NDO.

In Figure 5, we further show the tracking error with respect to the time for the step input. From the figure we can observe that the tracking error tends to zero after the transient phase, which confirms again the accuracy and robustness of our controller.

The next simulation shows the ability to follow a slow changing reference value represented by a sinusoidal input. For this purpose and for evaluating the performance of the proposed controller, a sinusoidal trajectory-tracking problem was studied. The desired trajectory is given by $0.015 \sin (3 t)+$ 0.015 , and the simulation interval time was selected as $0.01 \mathrm{~s}$. Furthermore a nonlinear disturbance observer is presented to estimate the unknown exogenous disturbances. The simulation results for evaluation of the tracking performance without/with NDO are depicted in Figures 6 and 7, respectively.
From Figure 6, it is obvious that the tracking performance of the system using only the proposed controller without integrating the nonlinear disturbance observer (NDO) is not satisfactory when there are modeling uncertainties and perturbations. On the other hand, as shown in Figure 7, the deviation between the actual and the desired trajectory is significantly reduced, when we integrate the NDO in the simulation system. The difference between the actual and the desired trajectory applying or not applying the nonlinear disturbance observer is highlighted by a comparison as depicted in Figure 8.

In Figure 9 a tracking error comparison between the desired and actual trajectory is illustrated. We can see that the tracking error with NDO tends asymptotically, with very small overshoot at the initial transients caused by the disturbances, to the zero. While the tracking error without NDO shows a number of fluctuations above the zero, this ensures again that the tracking performance or regulation obtained from the proposed controller (NDOBC) behaves better than the system without NDO.

As addressed earlier, it is hard to analyze the stability and tune the controller parameters when using PID controllers, in spite of the simplicity and the small number of parameters that they have to be adjusted. Therefore, a comparison has been conducted between NDOBC and conventional PID controller in Figure 10 to illustrate the advantage of our proposed Nonlinear Disturbance Observer Based Control (NDOBC) and its superior performance over the conventional PID controller.

\section{Conclusion}

The proposed Nonlinear Disturbance Observer Based Control law (NDOBC) provides a relatively new thread for the control of PMA. The advantage of this method not only is reflected in its high accuracy, but also manifests itself in convenience. The proposed approach represents a simple, yet robust, mechanism for guaranteeing finite time performance of zero error condition. The results of simulations demonstrate the validity of the proposed controller and show its superiority in tracking performance. In particular, the rapid response to parameter's changes and following of the desired trajectory are the deciding features. Based on the obtained results it is possible to realize the trajectory tracking control of pneumatic muscle (PM) pulling a mass against gravity practically.

\section{Conflict of Interests}

The authors declare that there is no conflict of interests regarding the publication of this paper.

\section{Acknowledgments}

This work was supported by the Natural Science Foundation of China under Grant 61075095, the International Science and Technology Cooperation Program of China "Precision Manufacturing Technology and Equipment for Metal Parts" under 
Grant no. 2012DFG70640, and the International Science and Technology Cooperation Program of Hubei Province "Joint Research on Green Smart Walking Assistance Rehabilitant Robot" under Grant no. 2012IHA00601.

\section{References}

[1] K. Xing, J. Huang, Y. Wang, J. Wu, Q. Xu, and J. He, "Tracking control of pneumatic artificial muscle actuators based on sliding mode and non-linear disturbance observer," IET Control Theory and Applications, vol. 4, no. 10, pp. 2058-2070, 2010.

[2] Y. M. Elobaid, J. Huang, Y. Wang, and J. Wu, "Tracking control of pneumatic muscle using Lyapunov based control method," in Proceedings of the International Conference on Modelling, Identification and Control (ICMIC '12), pp. 223-227, 2012.

[3] T.-Y. Choi and J.-J. Lee, "Control of manipulator using pneumatic muscles for enhanced safety," IEEE Transactions on Industrial Electronics, vol. 57, no. 8, pp. 2815-2825, 2010.

[4] D. G. Caldwell, G. A. Medrano-Cerda, and M. Goodwin, "Control of pneumatic muscle actuators," IEEE Control Systems Magazine, vol. 15, no. 1, pp. 40-48, 1995.

[5] D. G. Caldwell, N. Tsagarakis, and G. A. Medrano-Cerda, "Bio-mimetic actuators: polymeric pseudo muscular actuators and pneumatic muscle actuators for biological emulation," Mechatronics, vol. 10, no. 4-5, pp. 499-530, 2000.

[6] D. W. Repperger, K. R. Johnson, and C. A. Phillips, "Nonlinear feedback controller design of a pneumatic muscle actuator system," in Proceedings of the American Control Conference (ACC '99), pp. 1525-1529, San Diego, Calif, USA, June 1999.

[7] D. B. Reynolds, D. W. Repperger, C. A. Phillips, and G. Bandry, "Modeling the dynamic characteristics of pneumatic muscle," Annals of Biomedical Engineering, vol. 31, no. 3, pp. 310-317, 2003.

[8] L. Zhang, H. Gao, and O. Kaynak, "Network-induced constraints in networked control systems-a survey," IEEE Transactions on Industrial Informatics, vol. 9, pp. 403-416, 2013.

[9] K. Xing, Q. Xu, J. He, Y. Wang, Z. Liu, and X. Huang, "A wearable device for repetitive hand therapy," in Proceedings of the 2nd Biennial IEEE/RAS-EMBS International Conference on Biomedical Robotics and Biomechatronics (BioRob '08), pp. 919923, October 2008.

[10] H. Gao, T. Chen, and J. Lam, "A new delay system approach to network-based control," Automatica, vol. 44, no. 1, pp. 39-52, 2008.

[11] A. Hildebrandt, O. Sawodny, R. Neumann, and A. Hartmann, "A cascaded tracking control concept for pneumatic muscle actuators," in Proceedings of the European Control Conference, pp. 345-350, 2003.

[12] K. Xing, J. Huang, J. He, Y. Wang, Q. Xu, and J. Wu, "Sliding mode tracking for actuators comprising pneumatic muscle and torsion spring," Transactions of the Institute of Measurement and Control, vol. 34, no. 2-3, pp. 255-277, 2012.

[13] J. Huang, Z.-H. Guan, T. Matsuno, T. Fukuda, and K. Sekiyama, "Sliding-mode velocity control of mobile-wheeled invertedpendulum systems," IEEE Transactions on Robotics, vol. 26, no. 4, pp. 750-758, 2010.

[14] H. Zhang, H. Yan, F. Yang, and Q. Chen, "Quantized control design for impulsive fuzzy networked systems," IEEE Transactions on Fuzzy Systems, vol. 19, no. 6, pp. 1153-1162, 2011.
[15] Y. Manai and M. Benrejeb, "Robust stabilization for continuous Takagi-Sugeno fuzzy system based on observer design," Mathematical Problems in Engineering, vol. 2012, Article ID 836814, 18 pages, 2012.

[16] H. Zhang, H. Yan, T. Liu, and Q. Chen, "Fuzzy controller design for nonlinear impulsive fuzzy systems with time delay," IEEE Transactions on Fuzzy Systems, vol. 19, no. 5, pp. 844-856, 2011.

[17] E. Todorov, C. Hu, A. Simpkins, and J. Movellan, "Identification and control of a pneumatic robot," in Proceedings of the 3 rd IEEE RAS and EMBS International Conference on Biomedical Robotics and Biomechatronics (BioRob '10), pp. 373-380, September 2010.

[18] Z. Varga and M. Moučka, "Mechanics of pneumatic artificial muscle," Journal of Applied Science in the Thermodynamics and Fluid Mechanics, vol. 3, no. 2, p. 6, 2009.

[19] J. S. R. Jang, C. T. Sun, and E. Mizutani, "Neuro-fuzzy and soft computing-a computational approach to learning and machine intelligence," IEEE Transactions on Automatic Control, vol. 42, pp. 1482-1484, 1997.

[20] Z. Z. S. H. C. Yan, H. Zhang, and F. W. Yang, "Observer-based $\mathrm{H}$-infinity control for discrete-time stochastic systems with quantization and random communication delays," IET Control Theory and Applications, vol. 7, pp. 372-379, 2013.

[21] H. Zhang, Q. Chen, H. Yan, and J. Liu, "Robust $H_{\infty}$ filtering for switched stochastic system with missing measurements," IEEE Transactions on Signal Processing, vol. 57, no. 9, pp. 3466-3474, 2009.

[22] H. Schulte, "The characteristics of the McKibben artificial muscle, appendix H," in The Application of External Power in Prosthetics and Orthotics, National Academy of Sciences, National Research Council, Washington, DC, USA, 1961.

[23] G. K. Klute and B. Hannaford, "Accounting for elastic energy storage in McKibben artificial muscle actuators," Journal of Dynamic Systems, Measurement and Control, vol. 122, no. 2, pp. 386-388, 2000.

[24] J. J. E. Slotine and W. Li, Applied Nonlinear Control, vol. 199, Prentice-Hall, Englewood Cliffs, NJ, USA, 1991. 


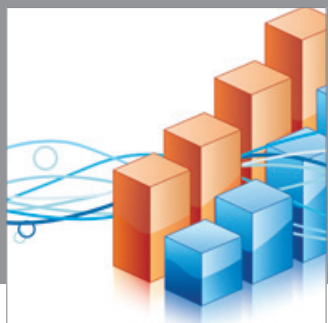

Advances in

Operations Research

mansans

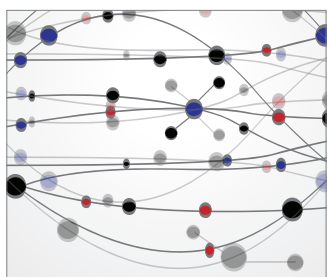

The Scientific World Journal
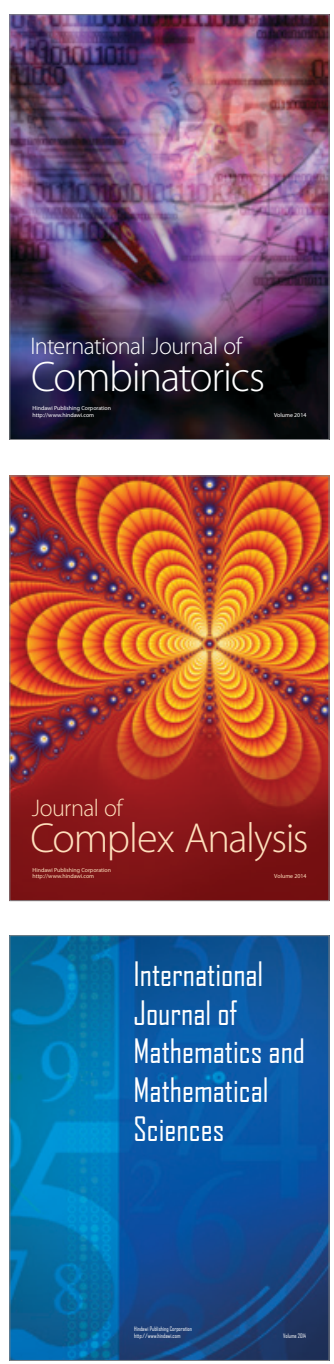
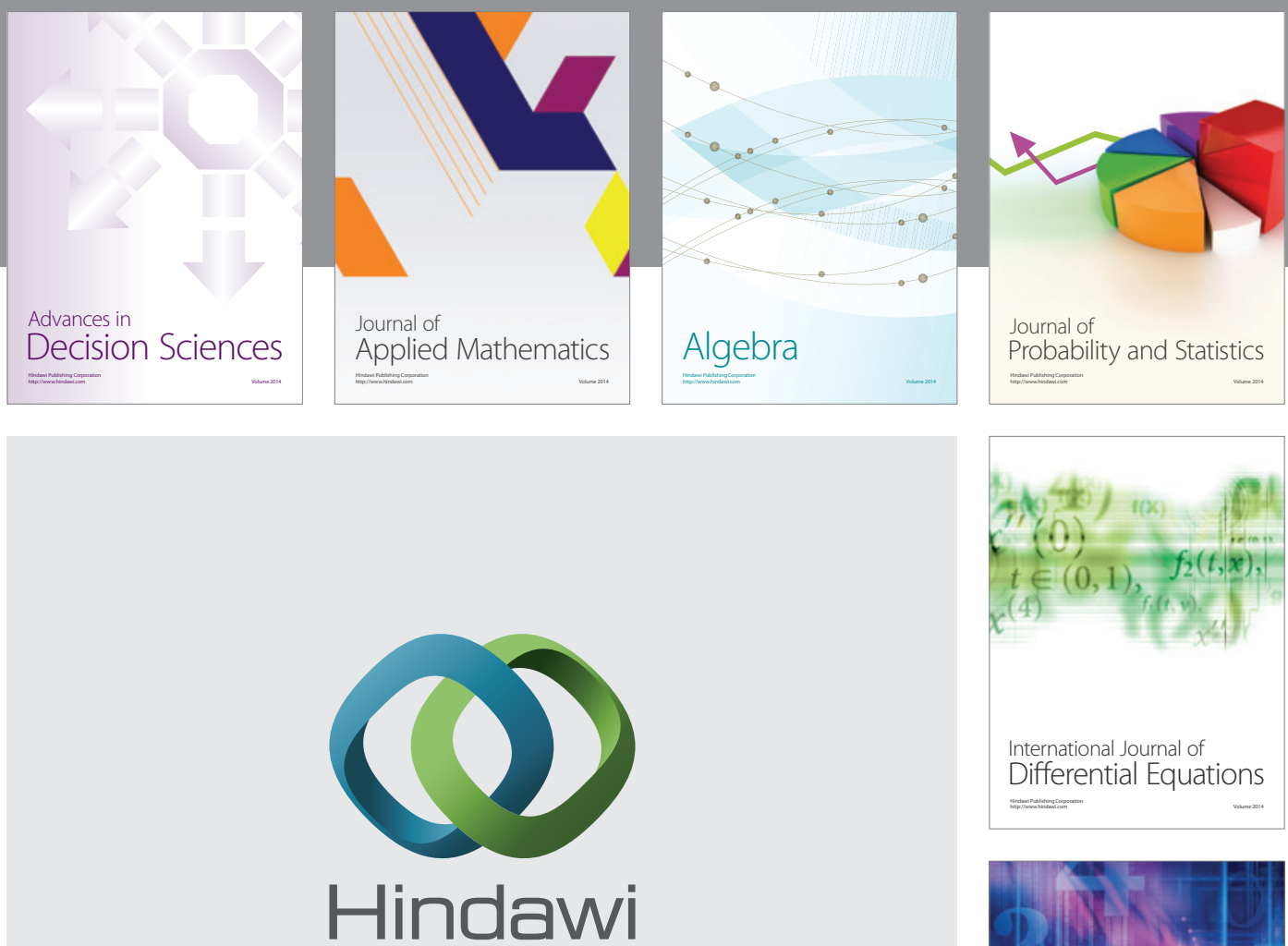

Submit your manuscripts at http://www.hindawi.com
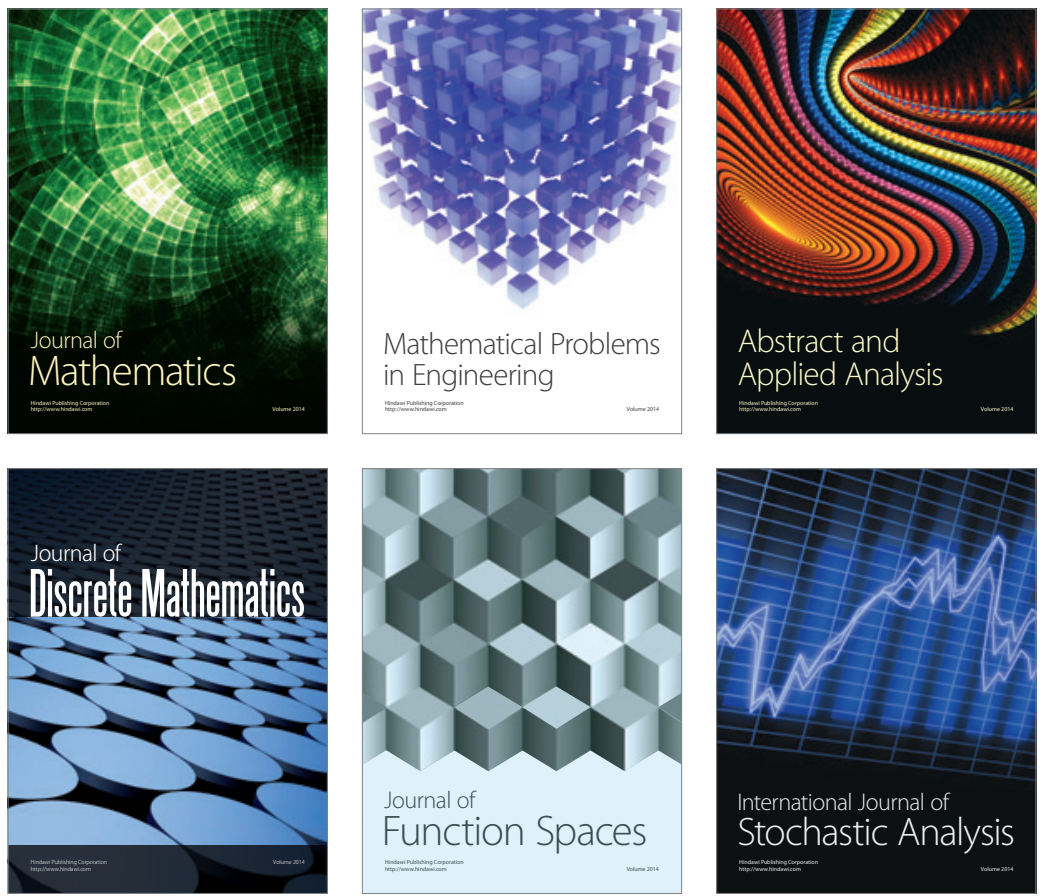

Journal of

Function Spaces

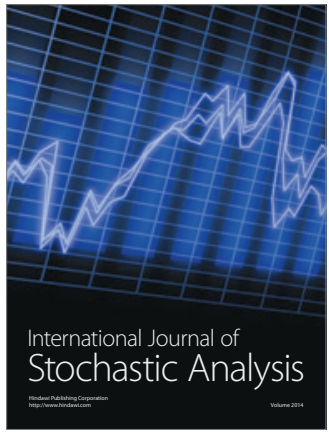

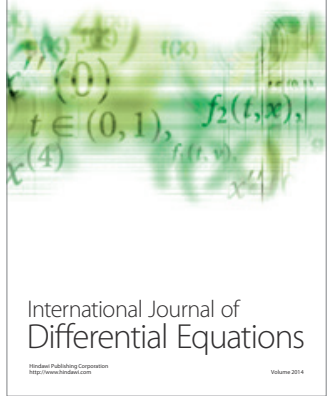
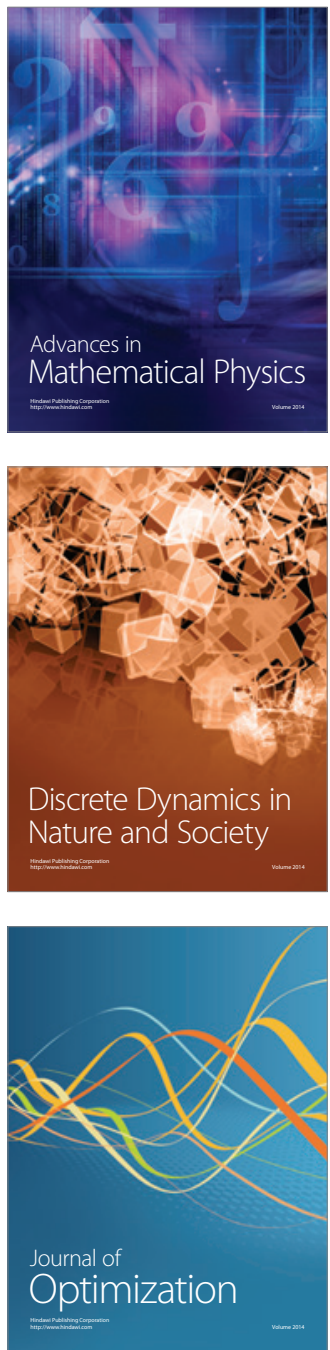